\begin{tabular}{|c|l|}
\hline Title & Photon emission at metal solution interface induced by electron injection from solvated electrons \\
\hline Author(s) & Murakoshi, Kei; U osaki, Kohei \\
\hline Citation & $\begin{array}{l}\text { Journal of V acuum Science \& Technology A: V acuum, Surfaces, and Films, 10(5), 2981-2984 } \\
\text { https://doi.org/10.1116/1.577896 }\end{array}$ \\
\hline Issue Date & $1992-09$ \\
\hline Doc URL & http://hdl.handle.net/2115/5970 \\
\hline Type & article (author version) \\
\hline File Information & JVS\&TA-V SF10-5.pdf \\
\hline
\end{tabular}

Instructions for use 


\title{
Photon emission at metal/solution interface induced by electron injection from solvated electrons
}

\author{
Kei Murakoshi and Kohei Uosaki \\ Physical Chemistry Laboratory, Department of Chemistry, Faculty of Science, Hokkaido University, \\ Sapporo 060, Japan
}

(Received 3 October 1991; accepted 30 December 1991)

\begin{abstract}
Light emission from metal, induced by electron injection from solvated electrons, was observed in an electrochemical system. The efficiency of emitted light increases drastically as electrode potential becomes positive. The high energy threshold of the spectrum increases as the electrode potential becomes positive by $1 \mathrm{eV} / \mathrm{V}$. The peak energy also shifts positively as the electrode potential becomes positive, although the degree of the shift is much smaller. Various processes are considered as a possible mechanism for a photon emission. From the qualitative comparison of the results at platinum and gold electrode, the contribution of the inverse photoemission process to the bulk band states at the light emission process in the electrochemical system is suggested. The feasibility of applying this phenomenon to a novel spectroscopy is discussed.
\end{abstract}

\section{INTRODUCTION}

Although many important processes take place at solid/ solution interfaces, e.g., electrochemical reaction, corrosion, and crystal growth, the understanding of these processes is still very immature. To fully understand how these processes proceed, one needs to know the electronic and morphological structures of solid surfaces and adsorbed molecules, i.e., reactants, intermediates, and products. Thus, the development of novel techniques to obtain the structural information of the solids and adsorbed molecules in situ is one of the most important subjects in the field of surface physical chemistry during the last decade. While in situ vibrational spectroscopic techniques provide the information of structures of adsorbed molecules ${ }^{1}$ and morphological structures of solid surfaces can be determined by an in situ electrochemical scanning tunneling microscope with atomic resolution, ${ }^{2}$ determination of surface electronic structure in situ is still very difficult in electrochemical systems. Although surface electronic states can be evaluated in ultrahigh vacuum by various techniques, e.g., ultraviolet photoemission spectroscopy (UPS) $^{3}$ and inverse photoemission spectroscopy (IPS), ${ }^{4}$ they are not suitable for an in situ investigation of solids in solution.

McIntyre and Sass proposed a new technique which may be able to determine the electronic structure of electrode. ${ }^{5}$ They observed light emission from a metal when highly energetic chemical species exist in solution. They considered that light is emitted as a result of radiative relaxation of energetic electrons injected from an electron donor in solution, e.g., an organic anion radical, to empty electronic states in a metal, i.e., inverse photoemission (IP) process, and, therefore, the spectrum of emitted light reflects the electronic structure of the metal. Ouyang and Bard confirmed the observation and supported this mechanism. They concluded that the relaxation is mainly to Schockley-type surface state. ${ }^{6}$

The quantitative analysis of the observed spectrum is, however, not easy because of the complexity both in the experimental procedure and in the interpretation. In the emission experiment proposed by McIntyre and Sass, the electrode potential is first pulsed to a very negative potential to generate the organic anion radical which acts as the electron injecting species, then it is pulsed to a positive potential so that the electron is injected from the anion radical to the metal. ${ }^{5}$ Usually this process is repeated many times to improve the $S / N$ ratio. During the potential pulsing, decomposition of solvent, supporting electrolyte, added organic compound and/or impurities may take place. Decomposition of these species results in electrogenerated chemiluminescence (ECL) which interferes the emission from metal. The decomposed species sometimes polymerize and deposit on the electrode surface ${ }^{7}$ and, therefore, reproducibility of the experiment becomes low. Furthermore, a large potential modulation causes reconstruction and roughing of the surface which also lowers the reproducibility. Thus, a new experimental procedure should be developed.

The interpretation of the results is not satisfactory either. Although it has been considered that IP is the only cause of the photon emission in an electrochemical system, the origin and nature of the states which participate to the radiation process are not clear. One reason for the difficulties of the quantitative analysis is the complex scattering process of the energetic electrons in the metal. Light emission from the metal induced by electron injection with excess energies of a few $\mathrm{eV}$ has been observed also at metalinsulator-metal (MIM), tunneling junction (TJ), ${ }^{8}$ and scanning tunneling microscope (STM) systems. ${ }^{9}$ A totally different model is proposed for the light emission at these systems. In these cases, it is considered that the tunneling electron excites a certain surface plasmon mode that decays radiately. ${ }^{8,9}$ Thus, it seems to be necessary to evaluate the various contributions to the light emission process in electrochemical systems to analyze the emitted photon spectrum.

In this work, we have used chemically generated solvated electrons as electron injecting species so that the potential of the electrode is always kept at relatively posi- 
tive potential region and decomposition of chemical species can be avoided during the emission measurement. We examined the origin of emitted light and discussed the feasibility of applying this method to determine electronic structure at the electrode/solution interface as a novel spectroscopy.

\section{EXPERIMENTAL}

Reagent grade hexamethylphosphoric triamide (HMPA: Wako Pure Chemicals Co. Ltd.) and sodium perchlorate (Aldrich Chemicals Co.) were used as solvent and supporting electrolyte, respectively. They were purified by usual manners ${ }^{10}$ and dehydrated in a vacuum. The electrolyte solution was prepared under a pure $\mathrm{N}_{2}(99.999 \%)$ atmosphere. Solvated electrons were generated by dissolving sodium metal (Wako Pure Chemicals Co. Ltd.) in 0.2 M $\mathrm{NaClO}_{4} / \mathrm{HMPA}$ solution. The prepared solution showed blue color with an absorption maximum at $770 \mathrm{~nm}$ which proves the formation of solvated electrons. ${ }^{11}$ Solvated electrons generated in this manner lasted for more than several hours at room temperature.

Metal electrodes ( $\mathrm{Au}$ and $\mathrm{Pt}$ ) were prepared on clean glass by vacuum deposition using a pure metal wire (99.99\%) as a source in $10^{-6}$ Torr vacuum. The temperature of the glass substrate during Au deposition was controlled by a hot-plate controller (Chino Co., DB-01-3) at $300^{\circ} \mathrm{C}$. The deposition rate and the thickness of the film were measured with a quartz crystal thickness monitor (ULVAC Co., CRTM-1000). Deposition rate $(0.1 \mathrm{~nm} / \mathrm{s}$ for $\mathrm{Au}$ and $0.01 \mathrm{~nm} / \mathrm{s}$ for $\mathrm{Pt}$ ) was controlled by changing the current passed through the tungsten wire around which metal wire was wound. Surface smoothness of the films was examined by scanning tunneling microscope (STM; Digital Instruments Co., NanoScope I).

Emission experiments were performed with a flow-type thin layer spectroelectrochemical cell made of Teflon. ${ }^{12}$ The separation between the UV-quartz optical window and the working electrode was set to $100 \mu \mathrm{m}$ with a Teflon spacer to minimize the absorption of emitted light by the electrolyte solution itself. The electrolyte solution was transferred from the supply flask to the thin layer compartment of the cell through a Teflon tube by positive $\mathrm{N}_{2}$ pressure with constant flow rate so that solvated electrons around the metal electrode were not depleted. The potential of the working electrode was referred to the $\mathrm{Ag} / 0.01$ $\mathrm{M} \mathrm{AgNO}{ }_{3}$ reference electrode. No electrochemical reactions due to residual moisture or other impurities were detected within the potential region examined in the present study $(-3.4-0 \mathrm{~V})$.

Light intensity was monitored with a photomultiplier tube (PMT: Hamamatsu Photonics Co. Ltd., R636) and a photon counter (NF Electronics, LI-574). Spectra of emitted light from metal electrode were obtained by using an imaging spectrograph (Jobin Yvon, CP-200) and a multichannel detector (Hamamatsu Photonics Co. Ltd., IMDC3330) with an image intensifier. ${ }^{13}$ Light emission measurements were carried out while the potential of the working electrode was either kept constant or swept be-

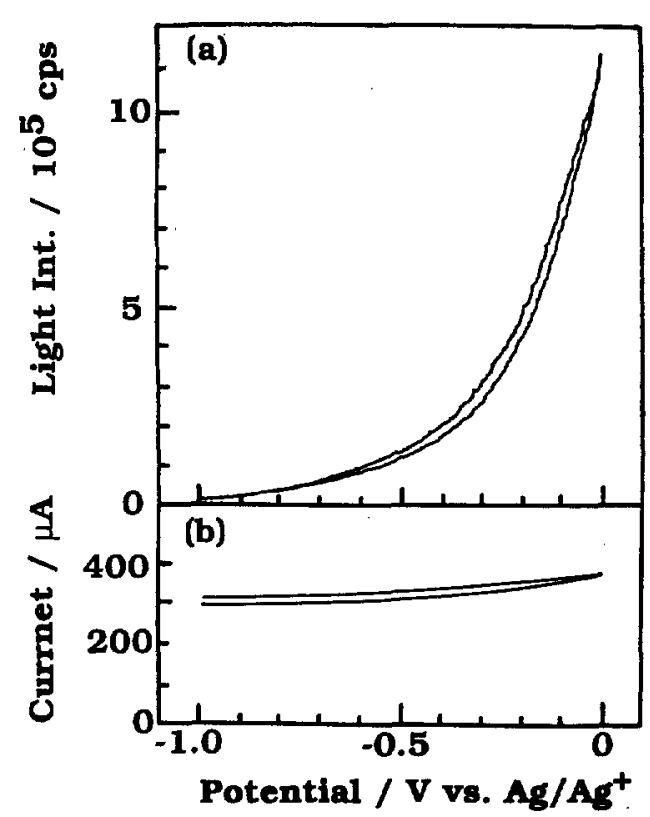

FIG. 1. Potential dependence of (a) emission intensity and (b) oxidation current at $\mathrm{Au}$ electrode in $0.2 \mathrm{M} \mathrm{NaClO} / \mathrm{HMPA}$ containing solvated electrons. Scan rate: $20 \mathrm{mV} / \mathrm{s}$

tween -1.0 and $0 \mathrm{~V}$. The positive potential limit $(0 \mathrm{~V})$ was chosen to avoid decomposition of the solvent.

All the measurements were carried out at room temperature.

\section{RESULTSS}

The intensity of emitted light from $\mathrm{Au}$ and oxidation current were recorded simultaneously as a function of electrode potential and typical results are shown in Fig. 1. Although the oxidation current increases only slightly as potential $U_{w}$ becomes positive, light intensity increases significantly. This means that the quantum efficiency of this process changes depending on electrode potential and is estimated as $10^{-8}$ at $0 \mathrm{~V}$ and $10^{-9}$ at $-1.0 \mathrm{~V} \cdot{ }^{14}$ The intensity of emitted light and anodic current did not change with time as long as electrode potential was kept constant.

Typical spectra of emitted light from $\mathrm{Au}$ are shown in Fig. 2. It is clearly seen that both the high energy threshold $E_{\mathrm{th}}{ }^{15}$ and the peak energy $E_{p}$ of the spectra increase as $U_{w}$ becomes more positive. Figure 3 shows the potential dependence of $E_{\mathrm{th}}$ and $E_{p} . E_{\mathrm{th}}$ shifts linearly with $U_{w}$ by 1 $\mathrm{eV} / \mathrm{V}$. The $E_{\mathrm{th}}$ is almost equal to the maximum energy of injected electron $E_{\text {inj }}$ which is given by the energy difference between the Fermi level of the metal and the highest electronic energy level of solvated electrons in solution, i.e., $E_{\mathrm{th}} / e=E_{\mathrm{inj}} / e=-\left(U^{0}-U_{w}\right)$, where $U^{0}$ is the redox potential of solvated electron. ${ }^{16,17}$ The potential dependence of $E_{p}$ is smaller than that of $E_{\mathrm{th}}$ and the shift is $\sim 0.2$ $\mathrm{eV} / \mathrm{V}$. Thus, the shape of the spectrum is also affected by the electrode potential (Fig. 2).

Photon emission was also observed at Pt electrode, although the intensity was much weaker. A typical spectrum is shown in Fig. 2. The potential dependence of the spectra 


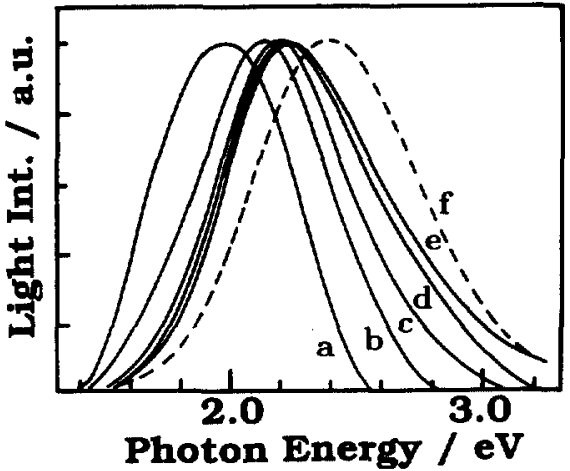

Fig. 2. Normalized spectra of emitted light from Au [(a)-(e);-] and Pt $[(\mathrm{f}) ;-.-$ ] electrodes in $0.2 \mathrm{M} \mathrm{NaClO} / 4$ HMPA containing solvated electrons. The electrode potential was kept at (a) $-0.8 \mathrm{~V}$, (b) $-0.6 \mathrm{~V}$, (c) $-0.4 \mathrm{~V}$, (d) $-0.2 \mathrm{~V}$, (e) $0 \mathrm{~V}$, and (f) $0 \mathrm{~V}$. The emission peak intensities are normalized to that of the spectrum (e). The enlargement factors are (a) 15.3 , (b) 4.4 , (c) 1.5 , (d) 1.2 , and (f) 1.1 , respectively.

at $\mathrm{Pt}$ has a tendency similar to that at $\mathrm{Au}$ as $E_{\text {th }}$ shifts by $1 \mathrm{eV} / \mathrm{V}$ and $E_{p}$ shifts to higher energy as electrode potential becomes positive. The $E_{p}$, however, is higher than that at $\mathrm{Au}$ by $0.1-0.2 \mathrm{eV}$ at a given potential when $U_{w}$ is more positive than $-0.2 \mathrm{~V}$ as shown in Fig. 3. When the $U_{w}$ is very negative, i.e., $E_{\mathrm{inj}}$ is very small, Spectra of $\mathrm{Pt}$ is very weak and it is very difficult to determine $E_{p}$ and $E_{\mathrm{th}}$. Therefore, only the values obtained at more positive potential than $-0.7 \mathrm{~V}$ were shown in Fig. 3.

\section{DISCUSSION}

There are various possibilities for the origin of the light emission at metal/electrolyte interfaces. The first possibility is the emission from chemical species in solution, e.g., ECL in which case the shape of spectrum is unique for a given system and should have no potential dependence. ${ }^{18}$ The linear relation between $E_{\mathrm{th}}$ and $U_{w}$ mentioned above

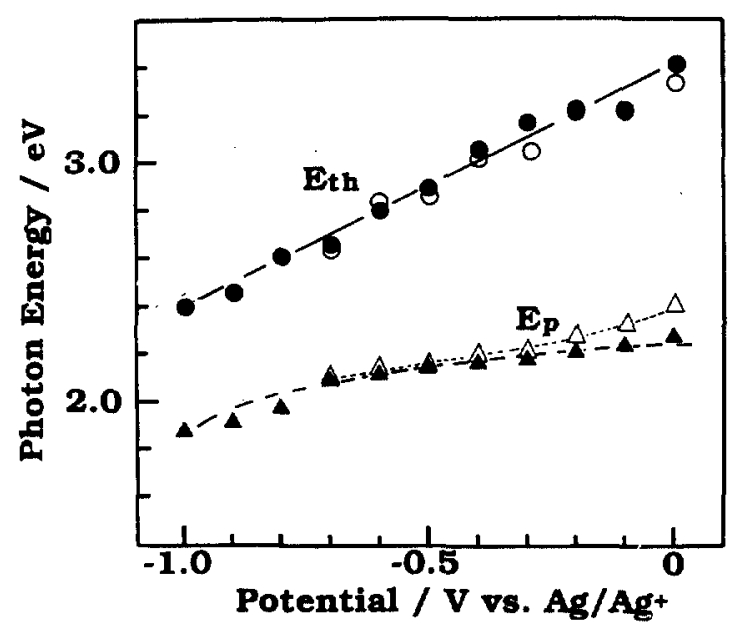

FIG. 3. Potential dependence of the high energy threshold $E_{\mathrm{th}}$ and the peak energy position $E_{p}$ of the spectra at $\mathrm{Au}(\mathbf{0}, \mathbf{\Delta})$ and $\mathrm{Pt}(0, \Delta)$ electrodes.

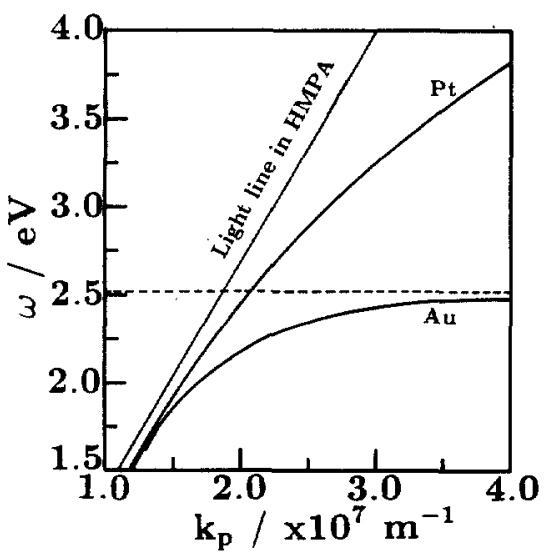

FIG. 4. Dispersion curves of surface plasmon at metal/HMPA interface.

excludes this possibility. Thus, it is certain that the light emission is from metal electrode induced by injected electrons.

The second possibility is a surface plasmon excitation decay process which is considered as the most important photon emission process in TJ and STM systems. ${ }^{8,9}$ At these tunneling junctions with a bias voltage of $2-5 \mathrm{~V}$, injected electrons induce photon emission as they excite surface plasmon which decays radiately. Surface plasmon is generally nonradiative but it becomes radiative when translational invariance along the surface is broken by roughness or irregularities at $\mathrm{TJ}^{8}$ or the presence of the microscope tip at STM. ${ }^{19}$ STM measurement showed that the surface of the electrode used here had relatively flat terrace of lateral dimension of a few tens $-100 \mathrm{~nm}$ which is a reasonable size for photons to couple with the surface plasmon mode around $2-3 \mathrm{eV} .{ }^{20}$ The total external quantum efficiency at $\mathrm{TJ}$ calculated by using the theory of Laks and Mills increases exponentially as bias voltage becomes larger. ${ }^{21}$ Similarly, the emission efficiency at STM system is enhanced as tip-sample voltage increases. ${ }^{9}$ In the present system, the quantum efficiency also increases drastically as $U_{w}$ becomes positive, i.e., $E_{\text {inj }}$ becomes larger. From the above considerations, the electromagnetic resonance at the metal surface seems to contribute for the photon emission process at the electrochemical system. Energy range of emitted photon can be estimated by the dispersion of surface plasmon at the metal/HMPA interface. The dispersion curves of Au and Pt in HMPA as well as light line in HMPA were calculated by using the tabulated dielectric constant $\epsilon_{m}$ and plotted in Fig. $4 .{ }^{22}$ Although the surface plasmon dispersion for $\mathrm{Pt}$ is drawn in Fig. 4, the resonance at Pt/HMPA interface is rather broad and strongly damped due to relatively large imaginary component of the dielectric constant of $\mathrm{Pt}^{23}$ The maximum of surface plasmon energy of Au is determined as $\omega$ for $k_{p} \rightarrow \infty$ and is indicated in Fig. 4 by dotted line. The surface plasmon excitation decay process can produce a spectrum of only photons with the energy lower than the maximum surface plasmon energy. The cut off energy at Au/HMPA interface is $\sim 2.5 \mathrm{eV}$ as shown in Fig. 4 and, therefore, the maximum energy of emitted photon is expected to $2.5 \mathrm{eV}$. 
However, the energy of emitted photons from Au always extends right up to $E_{\text {inj }}$ which can be much larger than 2.5 $\mathrm{eV}$ in some case. Furthermore, the photon emission was also observed at $\mathrm{Pt}$ electrode despite of the fact that the electromagnetic effect of surface plasmon does not operate due to the strong damping as mentioned before. Thus, the surface plasmon excitation decay process may be involved in the photon emission process but could not be the major contribution.

The other possible mechanism for the light emission process in this system is the charge transfer reaction inverse photoemission (CTRIP) process proposed by McIntyre and Sass. ${ }^{5}$ In this process, some of energetic electrons, injected from radical species in solution, relax radiately to unoccupied bulk or surface states at lower energies of the metal. Importance of surface states in CTRIP process was suggested by Ouyang and Bard ${ }^{6}$ and present authors. ${ }^{17}$ Electroreflectance (ER) spectroscopy study showed that the surface states of $\mathrm{Au}(111)$ are partly occupied at the potential of zero charge (pzc) in aqueous media $(-0.16$ $\mathrm{V}$ versus $\mathrm{Ag} / \mathrm{Ag}^{+24}$ ) and become unoccupied only when the electrode is biased sufficiently positive. ${ }^{25}$ The potential region examined in this study is relatively negative and it is reasonable to think that the surface states of $\mathrm{Au}$ are occupied and cannot contribute to the relaxation process. Furthermore, the surface state of $\operatorname{Pt}(111)$ is known to locate at higher energy than that of $\mathrm{Au}{ }^{26}$ Thus, if the spectrum of emitted light reflects the distribution of surface states, photons emitted from Pt should have smaller energy than that of $\mathrm{Au}$ at a given potential. However $E_{p}$ at $\mathrm{Pt}$ is almost the same as that at $\mathrm{Au}$ in the relatively negative potential region and shows more contribution of photons of higher energy in more positive potential region. These results suggest that the unoccupied electronic states of $\mathrm{Pt}$ to which injected electron relax are located at lower energy than that of Au contrary to the expectation. It seems, therefore, that the emitted photon spectrum reflects the relaxation of injected electrons to the bulk band states of metal rather than the surface states.

Large potential dependence of the emission intensity at the present system shown in Fig. 1 can be explained by considering the energy dependence of the radiative relaxation probabilities of electron in the spontaneous emission processes. It is known the probability becomes higher as the energy difference between the states is larger. ${ }^{27}$ This means that the emission efficiency should be higher as $E_{\text {inj }}$ becomes larger.

The above considerations suggest that the IP process to the bulk band states is the major contribution to the light emission from the metal in the present system. The spectrum reflects the density of the unoccupied states just above Fermi level and the relaxation probability of each transition. The spectrum of emitted light also contains the information of the electron transfer process, since the distribution of the injected electron energy was perturbed dur- ing the electron transfer process at an interface. More quantitative analysis of the spectra is under way to investigate these contributions.

\section{ACKNOWLEDGMENT}

This work was supported by a Grant-in-Aid for Scientific Research of the Ministry of Education, Science and Culture, Japan (02453001).

This article was presented at the 38th National Symposium of the American Vacuum Society Topical Conference, Surface Science at the Solid-Liquid Interface (TC1).

'Spectroelectrochemistry, edited by J. Gale (Plenum, New York, 1988). ${ }^{2}$ O. M. Magnussen, J. Hotlos, R. J. Nichols, D. M. Kolb, and R. J. Behm, Phys. Rev. Lett. 64, 2929 (1990).

${ }^{3}$ J. B. Pendry, Surf. Sci. 57, 679 (1976).

${ }^{4}$ N. V. Smith and D. P. Woodruff, Prog. Surf. Sci. 21, 295 (1986).

${ }^{5}$ R. McIntyre and J. K. Sass, Phys. Rev. Lett. 56, 651 (1986); J. Electroanal. Chem. 196, 199 (1985); R. McIntyre, D. K. Roe, J. K. Sass, and W. Storck, J. Electroanal. Chem. 228, 293 (1987); R. McIntyre, D. K. Roe, J. K. Sass, and H. Gerischer, in Electrochemical Surface Science, edited by M. P. Soriaga, ACS Symposium Series 378 (American Chemical Society, Washington, DC, 1988), p. 233.

${ }^{6}$ J. Ouyang and A. J. Bard, J. Phys. Chem. 91, 4058 (1987); 92, 5201 (1988).

'J. Ouyang and A. J. Bard, J. Electroanal. Chem. 222, 331 (1987).

${ }^{8}$ J. Lambe and S. L. McCarthy, Phys. Rev. Lett. 37, 923 (1976).

${ }^{9}$ J. H. Coombs, J. K. Gimzewski, B. Reihl, J. K. Sass, and R. R. Schlitter, J. Microsc. 152, 325 (1988); J. K. Gimzewski, J. K. Sass, R. R. Schlitter, and J. Schott, Europhys. Lett. 8, 435 (1989).

${ }^{10}$ D. D. Perrin, W. L. F. Armarego, and D. R. Perrin, in Purification of Laboratory Chemicals, 2nd ed. (Pergamon, New York, 1981).

"J. M. Brooks and R. R. Dewald, J. Phys. Chem. 72, 2655 (1968).

${ }^{12}$ K. Murakoshi and K. Uosaki, J. Electroanal. Chem. 308, 351 (1991).

${ }^{13}$ This system covers over a photon energy range of $1.4-3.5 \mathrm{eV}$. The resolution was $\sim 4 \mathrm{~nm}$ and the spectral response was calibrated by a tungsten-halogen lamp.

${ }^{14}$ The quantum efficiency of this process is estimated by assuming that $\sim 80 \%$ of photons are transmitted through electrolyte solution and quartz window, and the counting efficiency of the PMT is $-10 \%$.

${ }^{15}$ The high energy threshold $E_{\text {th }}$ was determined somewhat arbitrarily by extrapolating the high energy portion of the spectrum to abscissa (energy axis).

${ }^{16}$ From this relation, $U^{0}$ of solvated electrons is estimated as $-3.4 \mathrm{~V}$ vs $\mathrm{Ag} / \mathrm{Ag}^{+}$(Ref. 12).

${ }^{17}$ K. Uosaki, K. Murakoshi, and H. Kita, Chem. Lett. 1159 (1990); J. Phys. Chem. 95, 779 (1991).

${ }^{18}$ L. R. Faulkner, in Methods in Enzymology, edited by M. A. DeLuca (Academic, New York, 1978), Vol. 57, p. 455.

${ }^{19}$ P. Johansson, R. Monreal, and P. Apell, Phys. Rev. B 42, 9210 (1990).

${ }^{20}$ D. L. Mills, M. Weber, and B. Laks, in Tunneling Spectroscopy, edited by P. K. Hansma (Plenum, New York, 1982), p. 140.

${ }^{21}$ J. Kirtley, T. N. Theis, and J. C. Tsang, Phys. Rev. B 24, 5650 (1981), and references therein.

${ }^{22} \mathrm{The}$ dielectric response function of HMPA $\left(\epsilon_{s}\right)$ is $\sim 2.2$ (Ref. 23). The horizontal axis is the component of the wave vector parallel to the surface $k_{p}$ given by $k_{p}=(\omega / c)\left(1 / \epsilon_{m}+1 / \epsilon_{s}\right)^{-1 / 2}$.

${ }^{23}$ H. Ueba, Surf. Sci. 129, L267 (1983).

${ }^{24}$ F. Silva, M. J. Sottomayor, and A. Hamelin, J. Electroanal. Chem. 294, 239 (1990).

${ }^{25}$ S. H. Liu, C. Hinnen, C. N. V. Huong, N. R. D. Tacconi, and K. M. Ho, J. Electroanal. Chem. 176, 325 (1984).

${ }^{26}$ G. Larsson and P. O. Nilsson, Phys. Lett. A 85, 393 (1981).

${ }^{27}$ H. Eyring, L. J. Walter, and G. E. Kimball, in Quantum Chemistry (Wiley, New York, 1944), p. 114. 